\title{
Tumor-Infiltrating Immune Cell
}

National Cancer Institute

\section{Source}

National Cancer Institute. Tumor-Infiltrating Immune Cell. NCI Thesaurus. Code C153548.

Immune cells that have left the bloodstream and migrated into a tumor. 